\title{
Optofluidic lab-on-chips for high throughput 3D imaging of cells and tissues
}

\author{
Federico Sala, ${ }^{1 ; 2}$, Petra Paiè, ${ }^{l}$, Roberto Memeo, ${ }^{2}$, Roberto Osellame, ${ }^{1 ; 2}$, Andrea Farina, ${ }^{l}$, Andrea Bassi, ${ }^{2}$ and Francesca $^{2}$ \\ Bragheri $^{l}$ \\ ${ }^{1}$ Istituto di Fotonica e Nanotecnologie - Consiglio Nazionale delle Ricerche (IFN-CNR), 20133 Milano, Italy \\ ${ }^{2}$ Dipartimento di Fisica - Politecnico di Milano, 20133 Milano, Italy
}

\begin{abstract}
Optofludic lab-on-chips are compact microsystems that allow the manipulation, the analysis and the imaging of cells with high throughput even at the single cell level. The optofluidic microscopes on chip here presented are fabricated by femtosecond laser micromachining followed by chemical etching. The technique is ideally suited for rapid prototyping and allows 3D geometries and perfect alignment between microfluidic and optical components networks. The devices have been validated on cancer cell lines by imaging in three dimensions both clusters of cells growing on spheres and single cells.
\end{abstract}

\section{Introduction}

Recent advances in imaging technologies, as the enhancement of spatial and temporal resolution, push the limits of the research in biology by extending the imaging capabilities from the surface of cell samples deeper into organisms. However, one major challenge is to provide accurate statistical analysis that takes into account the heterogeneity of cellular behaviour in the same biological model.

Optofluidics provides a perfect synergy for the development of new imaging platforms able to perform recording and analysis of multiple samples with minimal photodamage, by the combination of microfluidics and optics. Optofluidic devices or lab-on-chip can thus be used for cell analysis and imaging either at the single cell level to study heterogeneity of specific cell properties in uniform populations or when cells during proliferation form a spheroid or even an organoid.

Three dimensional optical microscopy techniques, such as light sheet microscopy [1], have shown to be a powerful tool to rapidly image biological samples at high spatial resolution, nevertheless complex sample preparation and system alignment still limits the imaging of multiple specimen. Few integrated approaches have been proposed [2], and we recently demonstrated a selective plane microscope on-chip [3] that permits to analyse cellular spheroids at high throughput.

Here we report the fabrication and the experimental validation of a class of optofluidic light sheet fluorescent microscopes on-chip that exploit microfluidics for easy sample movements and laser light for the excitation of the samples under investigation. We show a new class of microscopes on chip where the optical and fluidic components have been designed to allow for dual-colour high throughput 3D imaging of cellular spheroids and single cells. The devices have been fabricated by femtosecond laser micromachining followed by chemical etching, which is ideally suited for rapid prototyping of optofluidic glass chip thanks to its 3D capability in irradiating both optical components as waveguides or lenses and microchannels. Laser writing is a serial fabrication technique that does not allow parallel fabrication, thus limiting the production yield. On the other hand it permits easily to modify the chip design in order to customize and optimize the devices. This feature has been exploited to realize microscope on chip for the $3 \mathrm{D}$ imaging of both tumour spheroids and single cancer cells.

\section{Device fabrication}

Femtosecond laser micromachining followed by chemical etching (FLICE) is exploited to fabricate both the optical and the fluidic components of the lab-onchips (LOCs). The technique is a two-step fabrication process consisting of: 1) permanent material modification due to nonlinear absorption of focused femtosecond laser pulses; 2) etching of the laser modified zone by a hydrofluoric acid (HF) solution. The laser irradiation enhances the etching rate in comparison to the pristine material, thus channels with arbitrary shape can be obtained in bulk glass substrates.

The integrated selective plane illumination microscope is composed by two embedded cylindrical lenses that are used to focus the laser light for imaging. Two light sheets that cross a C-shaped microchannel from two opposite sides are thus formed. This

Corresponding author: francesca.bragheri@ifn.cnr.it 
microchannel has a square cross-section and allows the sample scanning by letting the samples flow at a constant speed through the light sheets. The cylindrical lenses are realized with a microfluidic channel having an engineered cross-section to optimize aberrations and filled with a high refractive index fluid. A schematic of the device is shown in Fig.1 (left). The complicated microchannel network necessary to obtain both the lenses and the microchannel for sample delivery can be easily realized in a single irradiation step by exploiting the 3D capability of the FLICE technique.

Thanks to the versatility of FLICE technique, we have also fabricated prototypes where cylindrical lenses are empty and realized with the air/glass interface having an engineered profile to avoid aberrations of the focused light. The microchannel shape has also been modified, as the imaging of single cell requires a higher numerical aperture objective for the detection. A further optimization of the device has been introduced by inscribing in the substrate two waveguides facing the two lenses, which allows a precise alignment of the two light sheets in the microchannel. Filters have been also fabricated by FLICE in order to remove the light that is not coupled to the waveguide and to increase the signal to noise ratio of the illumination planes.

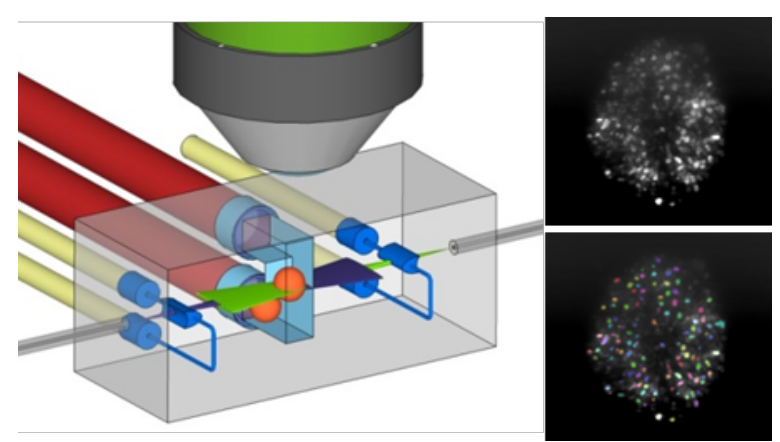

Fig. 1. Schematic of a dual-colour optofluidic selective plane illumination microscope on chip. An example of maximum intensity projection image of a tumour spheroid is shown on the right along with a segmentation of the image where the nuclei of cells are highlighted with different colours.

\section{Device characterization}

The engineering of the devices allowed obtaining different thicknesses of the light sheets ranging from 1.5 $\mu \mathrm{m}$ to $7 \mu \mathrm{m}$, which can be alternatively implemented on the chip on the basis of the sample to be analysed. For the microscope on chip validation fixed tissue spheroids of colon cancer cells expressing a histone H2B fluorescent nuclear reporter protein (mCherry) has been used. They were flown in the microchannel at a constant speed passing through the light sheet that excited fluorescence of a spheroid section at time. By collecting the emitted fluorescence the entire 3D image could then be reconstructed. An example of the Maximum Intensity Projection of a spheroid and its segmentation analysis are shown in Fig.1(right). The microfluidic microscope for single cell analysis has been validated with fixed breast cancer cells, demonstrating the capability of this device to obtain $3 \mathrm{D}$ reconstruction of the sample with very high resolution. High throughput acquisition is also demonstrated paving the way for a statistically significant method of single cell analysis by highresolution 3D imaging.

The results showed the possibility to obtain important biological insights in the modification of cellular properties during tumour progression. The increase in the achievable resolution would allow for imaging of the chromatin network so as to evidence modifications in the structure that might be associated to cancer features. All these advancement would open new possibilities towards the field of personalized medicine

This work was partially supported by the European Union under the FET Open PROCHIP project (grant agreement no. 801336).

\section{References}

1. J. Huisken, J. Swoger, F. Del Bene, J. Wittbrodt, E.H.K. Stelzer, Science 305, 1007 (2004)

2. P. Paiè, R. Martinez Vzquez, R. Osellame, F. Bragheri, A. Bassi, Cytometry Part A 93, 987 (2018)

3. P. Paiè, F. Bragheri, A. Bassi and R. Osellame, Lab on a Chip 16, 1556 (2016)

4. R. Osellame, H. J. W. M. Hoekstra, G. Cerullo, M. Pollnau, Laser \& Photonics Reviews 5, 442, (2011) 